\title{
Exciton Hamiltonian for the Bacteriochlorophyll System in the LH2 Antenna Complex of Purple Bacteria
}

\author{
Sergei Tretiak* \\ Theoretical Division, Los Alamos National Laboratory, Los Alamos, New Mexico 87545 \\ Chris Middleton, Vladimir Chernyak, and Shaul Mukamel* \\ Department of Chemistry, University of Rochester, P.O. RC Box 270216, Rochester, New York 14627-0216
}

Received: November 10, 1999

\begin{abstract}
An effective Frenkel-exciton Hamiltonian for the LH2 photosynthetic complex from Rhodospirillum molischianum is calculated using the collective electronic oscillator (CEO) approach combined with the crystal structure. The absorption spectra of the various bacteriochlorophyll aggregates forming the complex are computed using the CEO. Each electronic transition is further analyzed in terms of its characteristic electronhole motions in real space. Using a two-dimensional representation of the underlying transition density matrices, we identify localized and delocalized electronic transitions, test the applicability of the exciton model, and compute interchromophore electronic couplings. Förster energy-transfer hopping time scales within B800 and from the B800 to the B850 system, obtained using the computed coupling constants, are in excellent agreement with experiment.
\end{abstract}

\section{Introduction}

The primary processes of photosynthesis, the capture of sunlight and its subsequent conversion into chemical energy, constitute the very basis of all life. ${ }^{1,2}$ As one of the oldest methods that organisms have used to produce energy, evolution has been meticulously perfecting the photosynthesis process for billions of years. The high efficiency and flexibility of these processes, which take place in green plants, photosynthetic bacteria, and certain algae, make them an intriguing subject of study. ${ }^{3-8}$ The photosynthetic unit (PSU) is made of lightharvesting (LH) aggregates of the chlorophylls and carotenoids surrounding the reaction center (RC). Solar light is absorbed by the LH network, and the excitation energy is very efficiently transported to the RC where it is subsequently converted through a series of dark electron-transfer reactions into a stable charge separation. ${ }^{3-6,9}$ The overall energy-transfer conversion time scale is about 100 ps with $95 \%$ quantum efficiency. This organization of the photosynthetic system is common to both photosynthetic bacteria and higher plants.

The PSU of purple bacteria is the most studied and the best characterized among all known photosynthetic systems. Electron microscopy showed that the RC is located in the center of the LH1 aggregate, which is a circle made out of bacteriochlorophylls $a$ (Bchls- $a$ ) and carotenoids. ${ }^{10}$ This core antenna is surrounded by several smaller ring-shaped LH2 complexes. Some bacteria also have a third type, the LH3 complex. ${ }^{6}$ The energetic hierarchy leads to funneling of electronic excitations from LH3s (800 and $820 \mathrm{~nm}$ ) and LH2s (800 and $850 \mathrm{~nm}$ ) through LH1 $(875 \mathrm{~nm})$ to the RC. ${ }^{3-6,9} 8.5$ and $7 \AA$ electron microscopy projection maps of LH1 of Rhodospirillum rubrum ${ }^{11}$ and Rhodovulum sulfidophilum, ${ }^{12,13}$ respectively, have been reported. High-resolution $(2.5 \AA)$ crystal structures of the LH2 complex of two bacteria Rhodopseudomoas (Rps.) acidophila ${ }^{14}$ and Rhodospirillum (Rs.) molischianum ${ }^{15}$ are now available. The pigment structure of Rs. molischianum is shown in Figure 1.
The complex is an octameric circular aggregate of $\alpha \beta$ heterodimers (intrasubunits) where each unit noncovalently binds three Bchls- $a$ and one carotenoid (in addition the electrondensity map contains traces of a partially resolved second carotenoid). Bchls form two rings labeled by their absorption maxima. The upper B850 ring made of $16 \mathrm{Bchls}-a$ is represented by 8 pairs of $\alpha$ and $\beta$ Bchls- $a$, where each molecule is associated with corresponding $\alpha$ and $\beta$ polypeptides. The other eight Bchls- $a$ lie in a plane and form the lower B800 ring. A circle of eight carotenoids bridges the B850 and B800 rings; each carotenoid is in contact with one B800 and B850 Bchls- $a$. All relevant inter- and intraring $\mathrm{Mg}-\mathrm{Mg}$ distances are given in Table 3, and the molecular labeling scheme is shown in Figure 4. Rps. acidophila has a very similar nonameric structure. ${ }^{14}$ This high circular symmetry simplifies the computation of intermolecular interaction parameters and has triggered intensive theoretical modeling of numerous spectroscopic optical measurements including absorption, ${ }^{16-20}$ time-, frequency-, and polarization-dependent fluorescence, ${ }^{21}$ hole burning, ${ }^{22}$ pumpprobe, ${ }^{23-30}$ and three-pulse echoes. ${ }^{21,31}$ These provide microscopic probes for the organization and functionality of the PSU.

Energy migration in antenna complexes depends primarily on the electronic coupling between the donor and acceptor chromophores. For the weakly coupled B800-B800 and B800B850 molecules, energy transfer may be described ${ }^{4,32}$ by the Förster incoherent hopping mechanism, ${ }^{33}$ where the transfer rate (in $\mathrm{ps}^{-1}$ ) is given by $\mathrm{y}^{4,34}$

$$
k=1.18 J^{2} \Theta
$$

Here $J$ is the donor-acceptor electronic coupling (in $\mathrm{cm}^{-1}$ ), and $\Theta$ is the overlap integral between donor fluorescence and acceptor absorption lineshapes each normalized to a unit area on the $\mathrm{cm}^{-1}$ scale. On the other hand, B850-B850 couplings are strong, exceeding the energetic disorder, and the molecular exciton picture needs to be used for the description of energy 


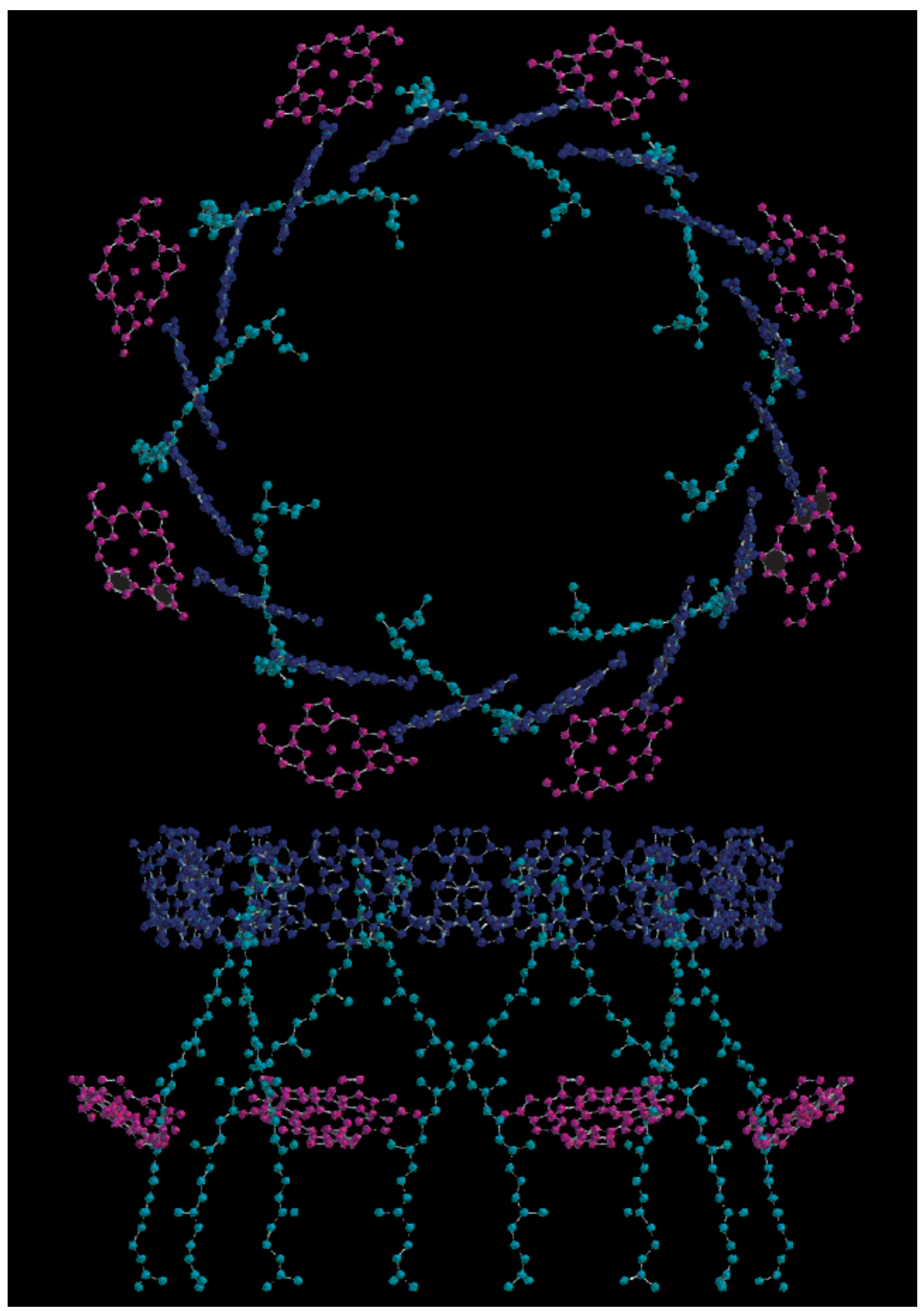

Figure 1. Top and side view of pigment in the light-harvesting complex II (LH2) of Rs. molischianum. The aggregate consists of the upper ring of 16 Bchls- $a$ (blue) paired in $8 \alpha \beta$ heterodimers or intrasubunits (B850 molecules), lower ring of 8 Bchl- $a$ (red) (B800 molecules), and ring of 8 lycopenes (carotenoids) (green).

migration in the upper B850 ring. ${ }^{35,36}$ In either case, the key parameters are the electronic couplings between chromophores.

If the chromophores are well separated (as in LH2), electron exchange is negligible: each molecule retains its own electrons, and interchromophore interactions are purely Coulombic. A point dipole approximation (PDA) is generally applied to compute this electrostatic interaction. The PDA further assumes that the chromophore sizes are small compared to their separation. The dipole-dipole coupling is then given by:4,37

$$
J=5042 \frac{\mu^{D} \mu^{A}}{R_{\mathrm{DA}}^{3} n^{2}}\left(\cos \alpha-3 \cos \beta_{1} \cos \beta_{2}\right)
$$

where $J$ is in $\mathrm{cm}^{-1}, \mu^{D}$ and $\mu^{A}$ are the transition dipole moments of donor and acceptor molecules in Debye, $R_{\mathrm{DA}}$ is the dipole separation in $\AA, n$ is the refractive index of the medium, $\alpha$ is the angle between dipoles, and $\beta_{1}$ and $\beta_{2}$ are the angles between each dipole and the vector connecting them.

Table 3 summarizes existing computations of LH2 couplings reported in the literature. Most calculations were performed for the Rps. acidophila complex (columns c-j); fewer results exist for the Rs. molischianum (columns a, b), whose crystal structure was reported more recently. The PDA that is routinely used for evaluating coupling constants does not hold for calculations of $J$ between closely lying chromophores such as in the LH2 system $^{32,38}$ since the chromophore sizes $(L \sim 9 \AA$ for Bchl- $a$ and $L \sim 27 \AA$ for carotenoid) are not small compared to their separations (see Table 3). More accurate computational techniques that can in principle take into account the Coulomb interaction between the actual charge distributions have been employed. ${ }^{16,32,39,40}$ The point-monopole approach that computes interaction between transition monopoles distributed on the atomic centers was used at the CIS level using the simplified PPP Hamiltonian. ${ }^{16}$ A different study evaluated the interaction between transition densities of each chromophore, which are calculated at the ab initio level with configuration interaction singles (CIS). ${ }^{32,38,68}$ This study found significant deviation from the PDA for the Rps. acidophila complex (compare columns d and e). However, these expensive ab initio/CIS calculations overestimate the excitation energies and the magnitudes of the 
transition dipole moments, and an overall scaling factor of 1.5, based on the ratio of the experimental to calculated dipole transition moments, was introduced in order to match the calculations with experiment. Despite the considerable numerical effort, the resulting couplings of $238 \mathrm{~cm}^{-1}$ (intradimer), 213 $\mathrm{cm}^{-1}$ (interdimer), and $25 \mathrm{~cm}^{-1}$ (B800-B800) are very similar to those obtained directly from the splittings in the dimer spectra reported in ref 38 (288, 221, and $28 \mathrm{~cm}^{-1}$, respectively). Excitonic couplings in Rs. molischianum were obtained by fitting effective Hamiltonian parameters to electronic spectra obtained with extensive semiempirical INDO/S/CIS calculations of the upper and lower rings. ${ }^{42,43}$ The cost of such calculations grows very rapidly with system size. The method has been applied to each ring separately, and evaluation of the interactions between the $\mathrm{B} 800$ and $\mathrm{B} 850$ rings, which requires computation of the entire LH2 aggregate, has not been reported yet. The INDO/S/CIS overestimates the porphyrin's transition dipoles by a factor of $\sim 2 .{ }^{43}$ This results in the larger than usual coupling parameters (column a). Electronic couplings (column j) were obtained recently from splittings in dimer spectra computed with INDO/S/CIS calculations of Bchl- $a$ dimers with nearest proteins from Rps. acidophila. ${ }^{40}$

In this article we apply the collective electronic oscillator (CEO) approach ${ }^{4,45}$ to study the electronic excitations of Bchl- $a$ aggregates of the LH2 complex of Rs. molischianum. This method is based on computing the optical response of small segments and avoids the quantum chemistry calculations of the whole complex. It provides an effective computational scheme for electronic excitations of large molecules. The optical response is calculated directly using equations of motion, and excited-state wave functions are not calculated explicitly. Analysis of the transition density matrices further allows one to visualize light-induced charge distribution and electronic coherences in real space. ${ }^{46-50}$ The electronic couplings are naturally obtained from these computations without invoking the $\mathrm{PDA}^{4,51}$ or similar approximations. Section II briefly describes the CEO method. In section III we analyze the electronic modes of the Bchl- $a$ monomers. In section IV we investigate the relevant electronic modes of the Bchl- $a$ dimers and link their properties to the corresponding modes of the monomers and to aggregate geometry. The Frenkel-exciton effective Hamiltonian is presented in section V. Finally we summarize our results and discuss the energy transfer rates in the LH2 complex in section VI.

\section{CEO Analysis of Electronic Excitations}

The numerical CEO-INDO/S procedure for calculating electronic structure has been described in detail elsewhere. ${ }^{44,45}$ The ZINDO code was first applied to generate the INDO/S Hamiltonian $^{52,55}$ using geometries obtained from crystal structures of the LH2 complex of Rs. molischianum with added hydrogen atoms. Geometries of hydrogen atoms of the substructures were optimized by using the semiempirical AM1 method. The nonhydrogen atoms were fixed to the crystal coordinates. We next calculated the Hartree-Fock ground-state density matrices ${ }^{56,57}$ that are the input to the following CEO calculation. The CEO procedure $^{44,45}$ was finally applied to compute the linearabsorption spectra and the relevant transition density matrices (denoted the electronic normal modes $\xi_{v}$ ), which connect the optical response with the underlying electronic motions. Each mode is a matrix representing the electronic transition between the ground state $|g\rangle$ and an electronically excited state $|v\rangle$. Its matrix elements are given by

$$
\left(\xi_{v}\right)_{m n}=\left\langle\nu\left|c_{m}^{+} c_{n}\right| g\right\rangle
$$

where $c_{m}^{+}\left(c_{m}\right)$ are creation (annihilation) operators of an electron at the $m$ th atomic orbital and $|g\rangle(|v\rangle)$ is the ground (excited)-state many-electron wave function. The modes are computed as eigenmodes of the linearized time-dependent Hartree-Fock (TDHF) equations of motion for the density matrix driven by the external field, totally avoiding the explicit calculation of many-electron excited-state wave functions. The optical transition frequencies are given by the eigenfrequencies $\Omega_{v}$ of these equations. ${ }^{44,45}$ The numerical effort involved in computing these eigenvalues and eigenvectors is greatly reduced by using the oblique Lanczos algorithm. ${ }^{58,59}$ All electronic transition frequencies have been computed very accurately (the convergence criteria has been set to $10^{-2} \mathrm{~cm}^{-1}$ for eigenvalues of the Liouville operator), which is vital for calculations of electronic coupling constants. Transition dipole moments $\mu_{v}=$ $\operatorname{Tr}\left(\mu \xi_{v}\right)$ were then calculated using the dipole moment operator $\mu=\sum_{n m} \mu_{n m} c_{m}^{+} c_{n}$, and $f_{v}=2 \Omega_{v} \mu_{v}^{2}$ is the oscillator strength of the $g$ to $v$ transition.

The electronic modes $\xi_{v}$ represent collective correlated motions of electrons and holes and carry substantially less information than the many-electron eigenstates but more than required for calculating molecular polarizabilities and spectroscopic observables. The diagonal element $\left(\xi_{v}\right)_{n n}$ represents the net charge induced on the $n$th atomic orbital by an external field with frequency $\Omega_{v}$, whereas $\left(\xi_{v}\right)_{m n}, n \neq m$, is the dynamical bond order (coherence) representing the joint amplitude of finding an electron on orbital $m$ and a hole on orbital $n$.

The INDO/S Hamiltonian uses four orbitals $\left(s, p_{\mathrm{x}}, p_{\mathrm{y}}\right.$, and $p_{\mathrm{z}}$ ) for each heavy atom of the LH2 complex. Instead of sorting out the $\pi$-electron contributions, we have used the following contraction. The total induced charge on each atom $\mathrm{A}$ is given by the diagonal elements

$$
\left(\xi_{v}\right)_{\mathrm{A}}=\sum_{n_{\mathrm{A}}}\left(\xi_{v}\right)_{n_{\mathrm{A}} n_{\mathrm{A}}}
$$

whereas an average over the off-diagonal elements represents the effective coherence between atoms $\mathrm{A}$ and $\mathrm{B}$

$$
\left(\xi_{v}\right)_{\mathrm{AB}}=\sqrt{\sum_{n_{\mathrm{A}} m_{\mathrm{B}}}\left[\left(\xi_{v}\right)_{n_{\mathrm{A}} m_{\mathrm{B}}}\right]^{2}}
$$

Here the indexes $n_{\mathrm{A}}$ and $m_{\mathrm{B}}$ run over atomic orbitals localized on atoms $\mathrm{A}$ and $\mathrm{B}$, respectively. The size of the matrix $\left(\xi_{v}\right)_{\mathrm{AB}}$ is now equal to the number of atoms. Two-dimensional representation of the electronic modes $\left(\xi_{v}\right)_{\mathrm{AB}}$ allows one to interpret and visualize these collective electronic motions in terms of the electronic density matrix in real space. ${ }^{46-50}$ The coordinate axes label atoms as given, e.g., in panel A of Figure 2 , where hole and electron positions are shown along the $x$ and $y$-axes, respectively.

\section{Bchl- $a$ Monomer}

Panel A of Figure 2 displays the $\beta$ B850 molecule. The computed linear absorption spectrum is shown in panel $\mathrm{B}$ where the oscillator strengths $f_{v}$ are plotted vs. transition frequencies $\Omega_{v}$. We use the standard labeling of active optical peaks for porphyrin-like systems $(\mathrm{Q}, \mathrm{B}, \mathrm{N})$. Additional transitions are 

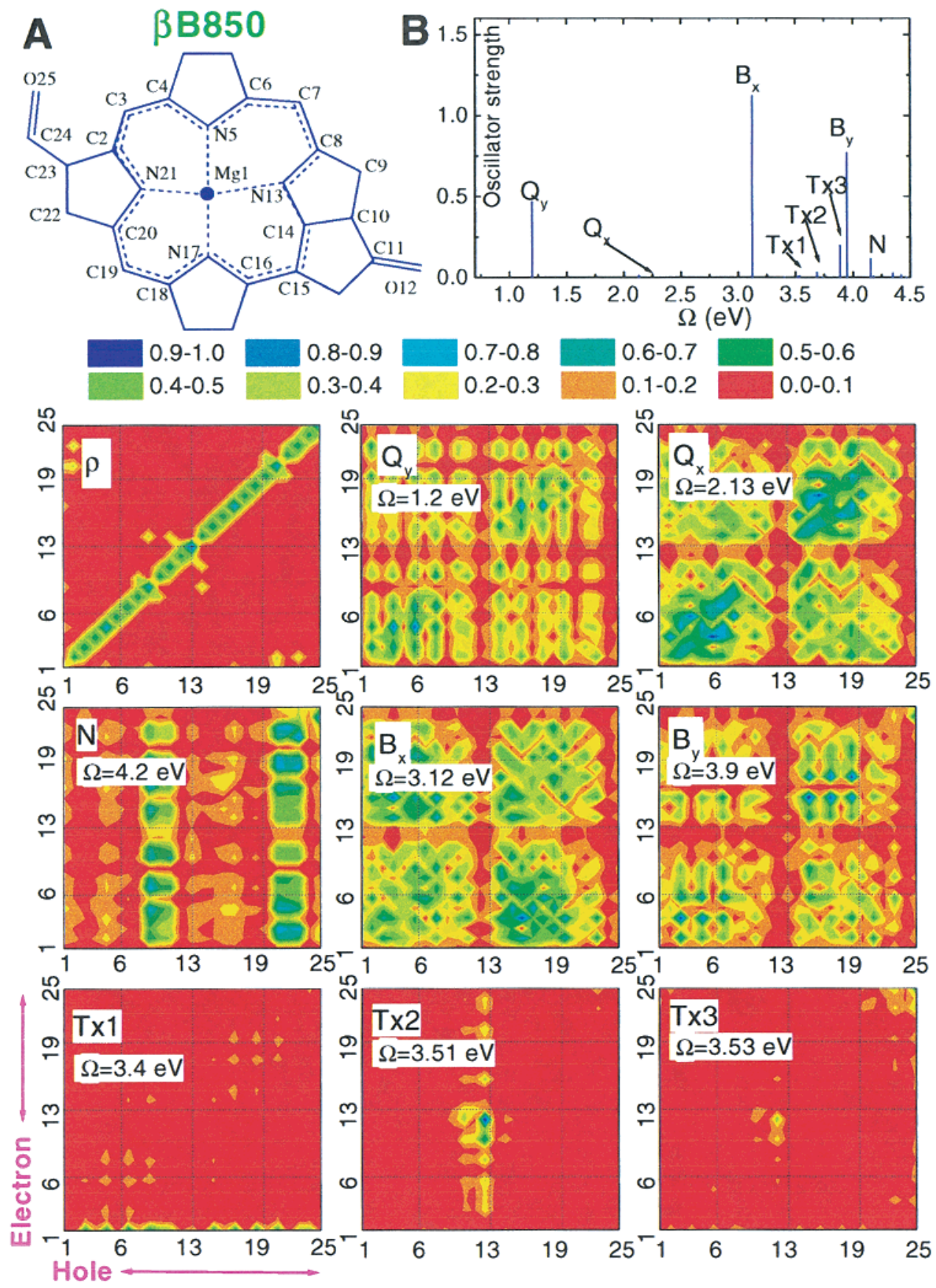

Figure 2. (A) Structure and atom labeling of Bchl- $a$. (B) Calculated linear absorption spectrum of Bchl- $a$. Contour plots the electronic modes that dominate the optical absorption of Bchl- $a$. The axis labels represent the individual atoms as labeled in panel A. The panels indicate the electronic mode according to panel B. The color code is given in the top row. Mode frequencies are given in Table 1.

TABLE 1: Calculated and Experimental Excitation Energies of $\beta$ B850, $\alpha$ B850, and B800 BChl- $a$ Molecules ${ }^{a}$

\begin{tabular}{lllll}
\hline & \multicolumn{1}{c}{$\alpha \mathrm{B} 850$} & $\mathrm{~B} 850$ & $\mathrm{~B} 800$ & experiment $^{b}$ \\
\hline $\mathrm{Q}_{y}$ & $1.20[1035](0.467)$ & $1.21[1026](0.481)$ & $1.21[1026](0.486)$ & $1.6[776]$ \\
$\mathrm{Q}_{x}$ & $2.13[583](0.015)$ & $2.12[586](0.010)$ & $2.16[575](0.007)$ & $2.16[575]$ \\
$\mathrm{B}_{x}$ & $3.12[398](1.122)$ & $3.09[402](1.089)$ & $3.11[400](1.034)$ & $3.17[392]$ \\
$\mathrm{Tx} 1$ & $3.40[365](0)$ & $3.34[372](0.006)$ & $3.66[339](0)$ & \\
$\mathrm{Tx} 2$ & $3.51[354](0.003)$ & $3.48[357](0.002)$ & $3.45[360](0.002)$ & \\
$\mathrm{Tx} 3$ & $3.53[352](0.009)$ & $3.36[370](0.007)$ & $3.97[420](0.016)$ & $3.47[360]$ \\
$\mathrm{B}_{y}$ & $3.90[318](0.970)$ & $3.87[321](0.993)$ & $4.29[290](0.010)$ & \\
$\mathrm{N}$ & $4.21[295](0.007)$ & $4.20[296](0.008)$ &
\end{tabular}

${ }^{a}$ Energies are in $\mathrm{eV}[\mathrm{nm}]$. Oscillator strengths are given in round parentheses. Molecular labeling is shown in Figure $4 .{ }^{b}$ Reference 60.

denoted Tx1-Tx3. The CEO calculations of Bchl- $a$ monomers ( $\alpha$ and $\beta$ B850 and B800) are presented and compared with experiment in Table 1. The experiments are for Bchl- $a$ monomers in ethyl ether solution. Monomers were extracted from Rs. Rubrum. Spectra of monomers from other species in different solvents are very similar.

The lowest $\mathrm{Q}_{y}$ transition carries a strong oscillator strength, which is vital for the light absorption function of the antenna, 
in contrast to the family of porphins where Q transitions are only weakly allowed. ${ }^{50}$ The Q-band in porphin is weak because the molecule is symmetric and the contribution of one (pyrrole + bridge) part cancels the dipole of the other part. This symmetry is broken in Bchl, and the Q-band consequently gains significant intensity. The computed $1.2 \mathrm{eV}$ frequency underestimates the experimental $(1.6 \mathrm{eV})$ value. This is generally the case in INDO/S/CEO calculations of porphyrins. ${ }^{50}$ However, the energy of the next $\mathrm{Q}_{x}$ state $(2.13 \mathrm{eV})$ matches the experiment $(2.16 \mathrm{eV}) . \mathrm{Q}_{x}$ is only weakly allowed in linear absorption. The following strong $\mathrm{B}_{x}$ and $\mathrm{B}_{y}$ peaks represent components of the Soret transition. The $\mathrm{B}_{x}$ frequency $(3.12 \mathrm{eV})$ is in excellent agreement with experiment $(3.17 \mathrm{eV})$, whereas that of $\mathrm{B}_{y}$ is significantly overestimated. This may be attributed to the three lower lying Tx transitions, which could be strongly coupled to $\mathrm{B}_{x}$ in the solvent, leading to an overall red-shift of this peak. (The experimental absorption of Bchl- $a$ in different solvents ${ }^{60}$ has a pronounced red-side shoulder on the $\mathrm{B}_{y}$ peak, which may be interpreted as one of the Tx transitions.) Finally, the highfrequency $\mathrm{N}$ transition is weak.

To trace the origin of the various peaks, we had examined the corresponding collective electronic modes. Two-dimensional plots of the matrices $\xi_{v}{ }^{45-49}$ establish a direct link between the optical response and the underlying photoinduced real-space dynamics of charges. The matrix size is equal to the number of heavy atoms, labeled according to panel A in Figure 2. We have only included atoms involved in the $\pi$-bonding, which are expected to dominate the UV/visible optical excitations.

Panel $\rho$ in Figure 2 shows that the ground-state density matrix of $\beta \mathrm{B} 850$ is pretty much diagonally localized, reflecting the nearest-neighbor chemical bonding in the ground state. The charge distribution along the diagonal is not uniform: nitrogen and oxygen atoms (blue dots on the diagonal) have an excess electronic charge. Panel $\mathrm{Q}_{y}$ displays the electronic mode of the lowest absorption peak $\mathrm{Q}_{y}$. This mode is delocalized across the entire molecule and is dominated by carbons $2-8$ and $15-20$. The coherences of the $\mathrm{Q}_{x}$ mode are distributed more uniformly across the molecule. The Soret $\mathrm{B}_{x}$ and $\mathrm{B}_{y}$ modes are very similar to the corresponding $\mathrm{Q}_{x}$ and $\mathrm{Q}_{y}$ transitions. Extensive delocalization and almost perfect symmetry with respect to the diagonal are common features of all Q and B modes. This reflects the absence of preferable direction of motion for holes or electrons. The $\mathrm{Mg}$ atom and both $\mathrm{C}=\mathrm{O}$ groups do not participate in these excitations, in contrast to the Tx modes, which heavily involve $\mathrm{Mg}$ and $\mathrm{C}=\mathrm{O}$ atoms. Panel $\mathrm{Tx} 1$ shows that the electron is transferred from the porphyrin to $\operatorname{Mg}(1)$ upon $\mathrm{Tx} 1$ excitation: the hole is delocalized ( $x$-axis) and the electron is localized on the $\mathrm{Mg}$ ( $y$-axis). This transition is forbidden in linear absorption for planar geometry, and its intensity grows as the $\mathrm{Mg}$ atom is displaced out of the molecular plane. Panel Tx2 shows that the next Tx mode represents electron transfer from the $\mathrm{O} 12$ oxygen mostly to $\mathrm{C} 11$ and to the rest of the molecule. As indicated earlier, oxygen is an electron acceptor that attracts extra electronic charge in the ground state. In the Tx2 excited state the electron gains energy and becomes more "loose". Similarly, the Tx 3 mode involves electron transfer from $\mathrm{O} 25$ to $\mathrm{C} 24$, with less electronic delocalization compared to Tx2. Finally, the $\mathrm{N}$ mode is localized on the two vertical strips and describes electron transfer from the pyrroles to the entire molecule. The $\mathrm{Q}, \mathrm{B}, \mathrm{N}$, and Tx electronic modes are very similar to the corresponding transitions in $\mathrm{Mg}$ and free-base porphins. ${ }^{50}$

Before turning to the dimers, we compare the electronic spectra of the various Bchl- $a$ monomers ( $\alpha$ and $\beta$ B850 and B800 molecules; see Table 1). Even though this is the same
Bchl- $a$, slightly different geometries in the crystal structure affect the spectra. The optically active transition frequencies $(\mathrm{Q}, \mathrm{B}$, and $\mathrm{N}$ ) do not change significantly (within $\sim 0.1 \mathrm{eV}$ ); however, the Tx energies change considerably. In particular we note that $\mathrm{Tx} 3$ is red-shifted from 3.53 to $2.96 \mathrm{eV}$ when going from $\beta \mathrm{B} 850$ to $\mathrm{B} 800$.

\section{Bchl- $a$ Dimer}

Panel A of Figure 3 displays the $\alpha \beta \mathrm{B} 850$ heterodimer (intrasubunit). The computed oscillator strengths $f_{v}$ are plotted vs transition frequencies $\Omega_{v}$ in panel B. Calculations of Bchl- $a$ dimers with the strongest electronic couplings are summarized in Table 2. The figure and the table clearly show that each monomer peak splits into two transitions in the dimer spectra (e.g., $\left.\mathrm{Q}_{y} \rightarrow \mathrm{Q}_{y} 1, \mathrm{Q}_{y} 2\right)$. The near-parallel orientation of the chromophores results in both $J$-type dimerizations (where the red component carries twice the oscillator strength whereas the blue component is dark, e.g., $\mathrm{Q}_{y}, \mathrm{~B}_{y}$ ), and the reverse H-type dimerizations (e.g., $\mathrm{B}_{x}$ ). This classification is common in molecular crystals and aggregated systems. ${ }^{61,62}$ The assignment of other dimer states is given in Table 2. We also observe two new transitions labeled CT1 and CT2, which are not associated with the monomer peaks.

To characterize the optical transitions, we analyzed the underlying electronic modes. Atom labeling runs over $\beta$ B850 first and then over $\alpha$ B850, following the pattern of Figure 2A. Panel $\rho$ in Figure 3 shows the ground-state density matrix of $\alpha \beta$ B 850. As expected, it is simply the superposition of the monomeric ground states (compare to panel $\rho$ in Figure 2), and both Bchls- $a$ are clearly identified. Panels $\mathrm{Q}_{y} 1$ and $\mathrm{Q}_{y} 2$ show a pair of states corresponding to the $\mathrm{Q}_{y}$ mode. The corners of the plot represent the monomers. They are separated by $\sim 9.2 \AA$ $\mathrm{Mg}-\mathrm{Mg}$ (3.7 $\AA$ between closest atoms) and completely uncoupled; i.e., there is no off-diagonal coherences between monomers. $\mathrm{Q}_{y} 1$ and $\mathrm{Q}_{y} 2$ are therefore symmetric and antisymmetric combinations, respectively, of the monomeric excitedstate wave functions. The interaction between monomers is purely electrostatic, which justifies using the Frenkel-exciton model for this aggregate. ${ }^{48,49,51}$ The $816 \mathrm{~cm}^{-1}$ splitting, which is a measure of electronic coupling between chromophores, provides the necessary information for constructing an effective Hamiltonian. ${ }^{51}$

Panels $\mathrm{Q}_{x} 1$ and $\mathrm{Q}_{x} 2$ show the next pair of modes. Compared with $\mathrm{Q}_{y}$, the interaction is very weak and results in $\sim 170 \mathrm{~cm}^{-1}$ splitting. This could be understood using the PDA model: the coupling in eq 1.2 depends on the square of the $\mathrm{Q}_{x}$ transition dipole, which is very small. Contrary to $\mathrm{Q}_{y}$, each $\mathrm{Q}_{x} 1$ and $\mathrm{Q}_{x} 2$ excited-state wave function is heavily dominated by a single monomer because of the small interaction and the difference in $\mathrm{Q}_{x}$ frequencies of $\alpha$ and $\beta$ monomers. Panels $\mathrm{B}_{x} 1$ and $\mathrm{B}_{x} 2$ display a pair of electronic modes corresponding to the $\mathrm{B}_{x}$ state. Similar to $\mathrm{Q}_{y}$, the strong transition dipoles of $\mathrm{B}_{x}$ result in the large $\sim 734 \mathrm{~cm}^{-1}$ splitting, and these states are again nearly symmetric and antisymmetric combinations of the excited-state monomer wave functions.

Modes CT1 and CT2 are completely different from the Q and $\mathrm{B}$ dimer states. They are delocalized over the off-diagonal regions, reflecting the electronic coherence between chromophores, and have no intramonomer contributions (diagonal regions). This explains the very weak oscillator strengths of these transitions. Upon CT1 excitation, the hole becomes localized on $\alpha \mathrm{B} 850$ ( $x$-axis), whereas the electron is transferred to $\beta \mathrm{B} 850$ ( $y$-axis). On the other hand, state CT2 describes electron transfer from $\beta$ to $\alpha$. Thus CT1 and CT2 correspond to intermolecular 


\section{A $\alpha \beta$ B850 Dimer}

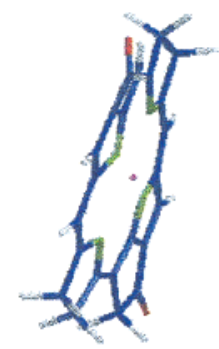

$\beta$
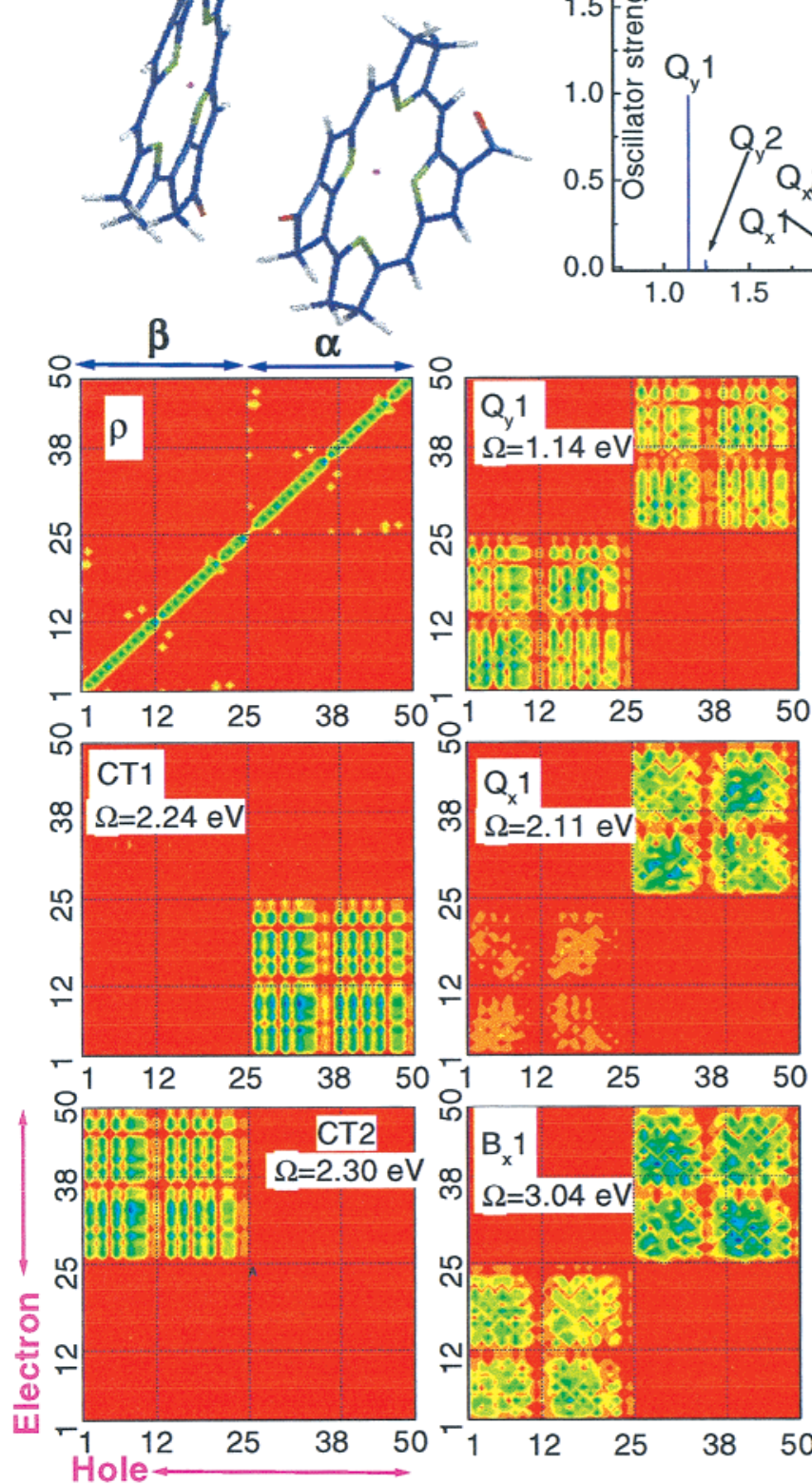
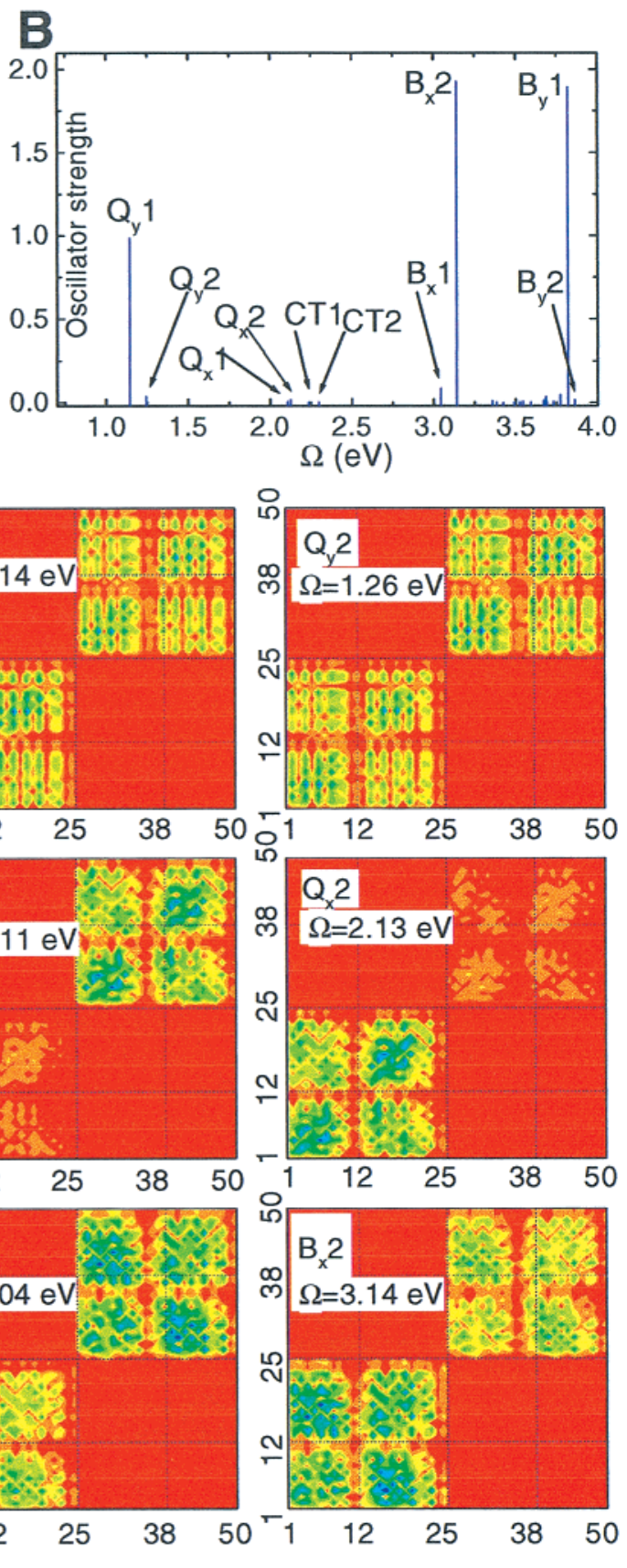

Figure 3. (A) Structure of the $\alpha \beta$ B850 dimer. (B) Calculated linear absorption spectrum of the $\alpha \beta$ B 850 dimer. Contour plots the electronic modes that dominate the optical absorption of the B850 dimer. The axis labels represent the individual atoms. Atoms of each monomer are labeled according to panel A of Figure 2. The panels indicate the electronic mode according to panel $\mathrm{B}$. The color code is given in Figure 2. Mode frequencies are given in Table 2.

charge-transfer excitations. These states lie just above the $\mathrm{Q}_{x}$ transitions and form a band that overlaps with the $\mathrm{Q}_{x}$ Frenkelexciton band in higher aggregates. Even though the CT modes are essentially forbidden in linear absorption, they show up in nonlinear optical studies of these aggregates (e.g., electroabsorption).

We have performed a similar analysis of the higher frequency $\mathrm{Tx} 1-\mathrm{Tx} 3$ and $\mathrm{B}_{y}$ transitions (see the second column of Table 2 ), but in the present paper we limit our discussion to the lowfrequency $\mathrm{Q}, \mathrm{B}_{x}$, and $\mathrm{CT}$ states that participate in the lightharvesting function of $\mathrm{LH} 2$.

Finally we compare several different Bchl- $a$ dimers with strong electronic couplings. The third column of Table 2 shows the $2 \alpha 1 \beta$ B850 aggregate (intersubunit). Since the intersubunit and intrasubunit aggregates are similar, the excitation energies and splittings of these dimers (second and third column) are very close. In contrast, a much weaker interaction is found in dimers $2 \alpha \mathrm{B} 850-1 \mathrm{~B} 800$ (upper-lower ring) and 2B800-1B800 (lower-lower ring) since the center-to-center separation of $\sim 25$ $\AA$ is larger compared to that of the neighboring molecules of the upper ring. Consequently the CT modes of these aggregates lie $\sim 1 \mathrm{eV}$ higher in energy (compare columns 2, 3 with columns 4, 5 of Table 2). Additional Bchl dimers and their electronic couplings will be discussed in the next section.

\section{Frenkel-Exciton Hamiltonian for $\mathrm{LH} 2$}

The CEO analysis of the dimer's electronic modes presented in the previous section shows that the interaction between 
TABLE 2: Calculated CEO Excitation Energies of Various Dimers Formed from $\alpha \mathbf{B 8 5 0}, \alpha \mathbf{B 8 5 0}$, and B800 Molecules ${ }^{a}$

\begin{tabular}{lllll}
\hline & $1 \alpha \mathrm{B} 850-1 \beta \mathrm{B} 850$ & $2 \alpha \mathrm{B} 850-1 \beta \mathrm{B} 850$ & $2 \alpha \mathrm{B} 850-1 \mathrm{~B} 800$ & $1 \mathrm{~B} 800-2 \mathrm{~B} 800$ \\
\hline $\mathrm{Q}_{y}-1$ & $1.145[1084.7](0.987)$ & $1.153[1077.2](0.897)$ & $1.204[1031.6](0.060)$ & $1.210[1026.4](0.842)$ \\
$\mathrm{Q}_{y}-2$ & $1.247[1000.0](0.038)$ & $1.244[998.4](0.098)$ & $1.217[1020.5](0.894)$ & $1.217[1020.5](0.139)$ \\
$\mathrm{Q}_{x}-1$ & $2.109[588.9](0.005)$ & $2.114[587.5](0.013)$ & $2.116[587.0](0.010)$ & $2.156[576.1](0.013)$ \\
$\mathrm{Q}_{x}-2$ & $2.130[583.1](0.017)$ & $2.115[587.2](0.002)$ & $2.156[576.1](0.007)$ & $2.156[576.1](0.002)$ \\
$\mathrm{C} 1$ & $2.238[555.0](0.002)$ & $2.105[590.0](0.006)$ & $3.100[400.6](0.018)$ & $3.155[393.7](0)$ \\
$\mathrm{CT} 2$ & $2.301[539.8](0.004)$ & $2.268[547.6](0.003)$ & $3.119[398.2](0.008)$ & $3.235[383.9](0)$ \\
$\mathrm{B}_{x}-1$ & $3.044[408.0](0.088)$ & $3.045[407.9](0.083$ & $3.089[402.1](0.800)$ & $3.112[399.1](0.215)$ \\
$\mathrm{B}_{x}-2$ & $3.142[395.3](1.927)$ & $3.144[395.0](1.925)$ & $3.111[399.2](1.298)$ & $3.114[398.8](1.858)$ \\
$\mathrm{Tx} 1-1$ & $3.383[367.1](0.003)$ & $3.371[368.4](0.009)$ & $3.358[369.9](0.005)$ & $3.664[339.0](0.007)$ \\
$\mathrm{Tx} 1-2$ & $3.426[362.5](0)$ & $3.406[364.7](0)$ & $3.666[338.8](0.010)$ & $3.665[338.9](0.006)$ \\
$\mathrm{Tx} 2-1$ & $3.491[355.8](0.002)$ & $3.471[357.8](0.002)$ & $3.450[360.0](0.002)$ & $3.443[360.7](0.002)$ \\
$\mathrm{Tx} 2-2$ & $3.527[352.1](0.003)$ & $3.542[350.6](0.003)$ & $3.482[356.7](0.001)$ & $3.452[359.8](0.002)$ \\
$\mathrm{Tx} 3-1$ & $3.360[369.6](0.012)$ & $3.353[370.4](0.012)$ & $2.958[419.9](0.016)$ & $2.953[420.6](0.016)$ \\
$\mathrm{Tx} 3-2$ & $3.544[350.5](0.007)$ & $3.513[353.5](0.011)$ & $3.342[371.6](0.008)$ & $2.961[419.5](0.015)$ \\
$\mathrm{B}_{y}-1$ & $3.844[323.1](1.895)$ & $3.854[322.3](1.880)$ & $3.872[320.8](0.475)$ & $3.963[313.4](1.760)$ \\
$\mathrm{B}_{y}-2$ & $3.862[321.6](0.018)$ & $3.866[321.3](0.083)$ & $3.936[315.5](1.535)$ & $3.971[312.8](0.263)$ \\
\end{tabular}

${ }^{a}$ Molecular labeling is shown in Figure 4. These dimers have the strongest B850-B850, B850-B800, and B800-B800 couplings. Energies are in $\mathrm{eV}[\mathrm{nm}]$. Oscillator strengths are given in round parentheses.

TABLE 3: Interchromophore Couplings $\left(\right.$ in $\left.\mathrm{cm}^{-1}\right)$ Calculated for $\mathrm{LH}^{a}$

\begin{tabular}{|c|c|c|c|c|c|c|c|c|c|c|c|c|c|}
\hline & $\mathrm{Mg}-\mathrm{Mg}$ & $\mathrm{CEO}\left(\mathrm{B}_{x}\right)$ & $\mathrm{CEO}\left(\mathrm{Q}_{y}\right)$ & $a$ & $b$ & $c$ & $d$ & $e$ & $f$ & $g$ & $h$ & $i$ & $j$ \\
\hline \multicolumn{14}{|c|}{ B850-B850 } \\
\hline $1 \alpha-1 \beta$ & 9.2 & 367 & 408 & 806 & 339 & 322 & 238 & 367 & 291 & 410 & 394 & 300 & 622 \\
\hline $2 \alpha-1 \beta$ & 8.9 & 369 & 366 & 377 & 336 & 288 & 213 & 284 & 273 & 310 & 317 & 233 & 562 \\
\hline $2 \alpha-1 \alpha$ & 18.0 & 61 & -102 & -152 & & & -46 & -48 & -50 & & & & \\
\hline $2 \alpha-1 \beta$ & 17.4 & 58 & -63 & & & & -37 & -37 & -36 & & & & \\
\hline $1 \alpha-2 \beta$ & 25.6 & 23 & 31 & & & & & & 12 & & & & \\
\hline $3 \alpha-1 \beta$ & 25.9 & 20 & 30 & & & & & & 12 & & & & \\
\hline \multicolumn{14}{|c|}{ B800-B850 } \\
\hline $1-1 \alpha$ & 25.5 & 6.1 & 14 & & 3.8 & -11.3 & -13 & -12 & -12.6 & 7 & -8 & & \\
\hline $1-1 \beta$ & 20.1 & 32 & 40 & & 15.7 & 4.8 & 5 & 4 & -3.8 & 6 & -2 & & \\
\hline $1-2 \alpha$ & 19.2 & 6.9 & -52 & & -22.7 & 25.7 & 27 & 27 & 27 & 29 & 16 & & \\
\hline $1-2 \beta$ & 22.8 & -23 & 10 & & 2.9 & 6.1 & 23 & 31 & 12 & 13 & 4 & & \\
\hline \multicolumn{14}{|c|}{ B800-B800 } \\
\hline $1-2$ & 22.0 & 7.0 & -25 & & -14 & -22 & -27 & -26 & 25 & & -15 & & \\
\hline $1-3$ & 40.7 & 2.3 & -3.5 & & & & -3 & -3 & & & & & \\
\hline
\end{tabular}

${ }^{a}$ The pigments are labeled according to Figure 4 . The second column shows the $\mathrm{Mg}-\mathrm{Mg}$ distance $(\AA)$. The CEO results for $\mathrm{B}_{x}$ (third column) and $\mathrm{Q}_{y}$ (fourth column) transitions of Rs. molischianum are shown in Figure 4 as well. The table also summarizes couplings for the $\mathrm{Q}_{y}$ band reported in the literature for Rs. molischianum (columns $a, b$ ) and Rps. acidophila (columns $c-j$ ): $a$ References 42 and 43 . Semiempirical INDO/ CIS calculations of the whole upper ring and further spectral modeling of the results. $b$ Reference 4 . PDA calculations with $\mu^{2}=68 \mathrm{D}^{2} . c$ Reference 4. PDA calculations with $\mu^{2}=68 \mathrm{D}^{2} . d$ References 32 and 38. Ab initio molecular orbital calculations. Transition density cubes approach. $e$ Reference 32 and 38. PDA calculations with $\mu=6.13$ D. $f$ Reference 16. Point monopole approximation based on the SCMO-PPP-CI computations. $g$ Reference 34. PDA with $\mu^{2}=41 \mathrm{D}^{2}$. $h$ Reference 17. Semiempirical QCFF/PI quantum mechanical calculations. $i$ Reference 41. Modeling the absorption and CD spectra. $j$ Reference 40. INDO/S/CIS calculations of splittings in Bchls- $a$ dimer spectra.

chromophores is purely electrostatic except for the two chargetransfer transitions. Each monomer has a Davydov splitting ${ }^{63,64}$ in the dimer spectra. In fact, the excitation frequencies of the monomer and dimer transitions provide all information necessary to construct the effective Hamiltonian for this LH2 complex, using an approach previously developed and tested on phenylacetylene dendrimers and naphthalene dimers ${ }^{51}$ and on the Rps. acidophila $\mathrm{LH} 2$ complex. ${ }^{40}$

The Frenkel-exciton model for an assembly of two-level chromophores coupled via Coulomb interactions has a form ${ }^{63,65}$

$$
H=\sum_{n} \Omega_{n} B_{n}^{+} B_{n}+\sum_{n \neq m} J_{n m} B_{m}^{+} B_{n}
$$

Here $\mathrm{B}_{m}\left(\mathrm{~B}_{m}^{+}\right)$is the annihilation (creation) operator of an excitation localized on the $m$ th chromophore and $\Omega_{n}$ represents the transition energy from the ground state to the excited state on the $n$th chromophore. The hopping parameters $J_{n m}$ represent the Coulomb interaction between chromophores. These may be obtained by computing the excitation energies of the dimer built from the $m$ and $n$ chromophores by solving the eigenvalue problem

$$
\left(\begin{array}{ll}
\Omega_{n} & J_{n m} \\
\mathrm{~J}_{\mathrm{mn}} & \Omega_{m}
\end{array}\right)\left(\begin{array}{l}
\varphi_{\alpha}(n) \\
\varphi_{\alpha}(m)
\end{array}\right)=\epsilon_{\alpha}\left(\begin{array}{l}
\varphi_{\alpha}(n) \\
\varphi_{\alpha}(m)
\end{array}\right), \quad(\alpha=1,2)
$$

Here $\varphi_{\alpha}(n)\left(\varphi_{\alpha}(m)\right)$ and $\Omega_{n}\left(\Omega_{m}\right)$ are the monomeric excitedstate wave functions and their energies and $\epsilon_{\alpha}$ are the energies of the dimer states. The electronic coupling is computed from the solution of eq 5.2

$$
4 J_{\mathrm{nm}}^{2}=\Delta \epsilon^{2}-\Delta \Omega^{2}
$$

where $\Delta \epsilon=\epsilon_{2}-\epsilon_{1}$ is the electronic splitting between a pair of dimer states and $\Delta \Omega=\Omega_{n}-\Omega_{m}$. The energies $\epsilon_{2}\left(\epsilon_{1}\right)$ and $\Omega_{n}$ $\left(\Omega_{m}\right)$ are obtained by a direct CEO calculation of the $(m n)$ dimer and each separate monomer. The contribution from other electronic states to the dimeric splitting is negligible for wellseparated states. For identical chromophores we have $J_{n m}=|\Delta \epsilon| / 2$. On the other hand, if the chromophores are very different and $\Delta \epsilon \approx \Delta \Omega \gg J_{n m}$, the numerical accuracy of this procedure is significantly reduced since $\Delta \epsilon$ and $\Delta \Omega$ should be 
calculated with high accuracy. Furthermore, the contributions from other electronic states may not be neglected. These problems do not apply to LH2, which is made out of nearly identical Bchls, and this method is numerically fast and inexpensive. (CEO calculations take about $2 \mathrm{~h}$ to compute a single parameter of Hamiltonian from the corresponding dimer (up to $\mathrm{B}_{y}$ states pair). The timing results are for a single PII $400 \mathrm{PC}$ workstation. The memory requirements are minor $(<5$ $\mathrm{MB})$ ). Note that a single CEO computation of a dimer gives the electronic couplings for all excited-state pairs. The sign of the coupling is readily determined using the structure of dimer electronic modes. For negative $J$, the lower (upper) state is a symmetric (antisymmetric) combination of the monomer blocks (this is the $J$ aggregate geometry). This order is reversed for positive $J$ (known as $H$ aggregates).

By considering different dimers from the LH2 complex we have computed the effective Hamiltonians for the $\mathrm{Q}_{y}$ and $\mathrm{B}_{x}$ excitonic bands that dominate the linear response. These parameters are given in columns 3, 4 of Table 3 and compared with other calculations reported in the literature. The cartoons in Figure 4 graphically display the computed electronic couplings among different chromophores of the Rs. molischianum LH2 complex.

Let us examine the intermolecular interactions between $\mathrm{Q}_{y}$ states. As expected, closely spaced B850 molecules of the upper ring have a strong interaction. The intradimer coupling $(\sim 408$ $\left.\mathrm{cm}^{-1}\right)$ slightly exceeds its interdimer counterpart $\left(\sim 366 \mathrm{~cm}^{-1}\right)$. The interaction significantly decreases for next-nearest neighbors $\left(\sim 100 \mathrm{~cm}^{-1}\right)$, and further for next-nearest neighbors $(\sim 30$ $\left.\mathrm{cm}^{-1}\right)$. The signs of the couplings alternate with chromophore separation. We found all other couplings to be smaller than 10 $\mathrm{cm}^{-1}$. These results generally agree with couplings of the $\mathrm{Q}$ states computed using other approaches (Table 3). However, much higher intradimer interaction of $806 \mathrm{~cm}^{-1}$ has been reported in for Rs. molischianum. The reported couplings for Rps. acidophila (columns $\mathrm{c}-\mathrm{j}$ ) decrease faster with distance between chromophores in the upper ring.

The upper-lower ring interaction is considerably weaker. Each B800 pigment shows significant coupling only to four nearest molecules from the B850 ring. The computed B800B850 electronic couplings $\left(\sim 50 \mathrm{~cm}^{-1}\right)$ are markedly larger than other calculations (see Table 3). Finally, the interaction between neighboring B800 pigments is small and negative and practically vanishes for the next-to-nearest neighbor molecules. The $\mathrm{B}_{x}$ electronic couplings shown in the lower graph of Figure 4 are generally smaller than their $\mathrm{Q}_{y}$ counterparts and are always positive (except $1-2 \beta$ ). It is interesting to note that B850 interand intradimer couplings are almost identical for the $\mathrm{B}_{x}$ band. The space orientation of the $\mathrm{B}_{x}$ transition dipoles lead to the strong (weak) coupling of B800 with $\beta(\alpha)$ bacteriochlorophylls of B850.

\section{Discussion}

Understanding the photophysical function of the antenna requires detailed information on energetic disorder, protein relaxation, and energy-transfer time scales, spectral overlaps, etc. Most of these quantities may be extracted from femtosecond nonlinear optical studies, ${ }^{18,31}$ and the electronic coupling constants are the key ingredients for a successful modeling. 4,35 The accuracy of these parameters depends crucially on the ability to predict transition dipole moments. For example, ab initio/ CIS and QCFF/PI/CIS approaches significantly overestimate the dipoles, requiring introduction a rescaling factor $\mu_{\text {calc }} / \mu_{\exp }$ to calculate interactions. ${ }^{17,32,38}$ The semiemperical INDO/S/CIS
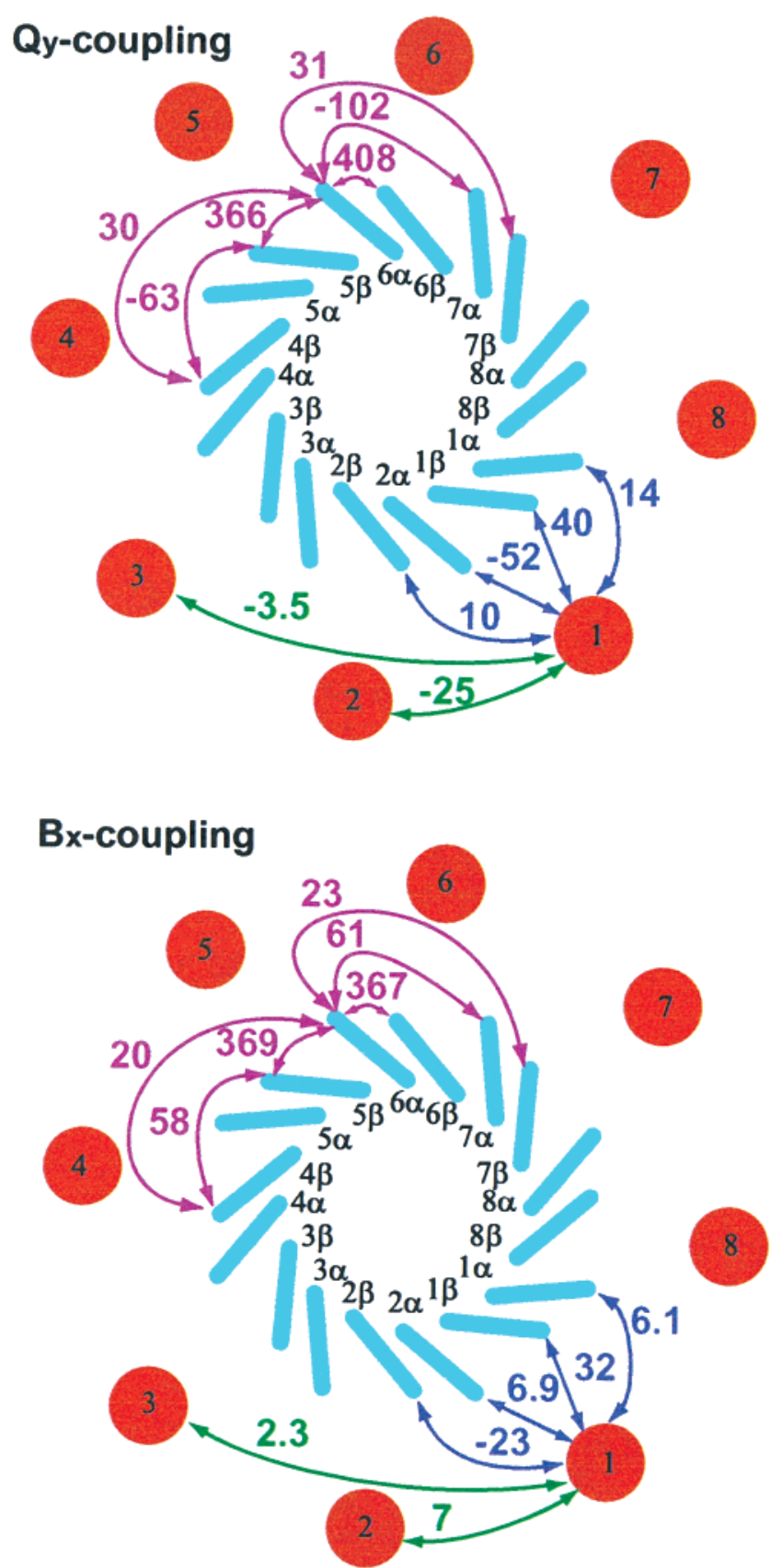

Figure 4. Graphical representation of the calculated excitonic couplings (in $\mathrm{cm}^{-1}$ ) of the LH2 complex shown in Figure 1. These couplings are also given in Table 3.

approach tends to overestimate transition dipole moments in porphyrin-like systems as well. ${ }^{66}$ This is probably the reason for the larger computed couplings $\mathrm{s}^{40,42,43}$ compared to other calculations. The CEO computed $\mathrm{Q}_{y}$ transition dipole moment of $\mathrm{Bchl}$ monomer is $1.44 e \cdot \AA$, which compares well with the experimental value of $1.33 e \cdot \AA .^{38}$ The difference may be attributed to vibronic coupling between $\mathrm{Q}_{y}$ and $\mathrm{Q}_{x}$ and to the effects of dielectric medium, which result in transfer some of the $\mathrm{Q}_{y}$ oscillator strength to $\mathrm{Q}_{x}{ }^{43}$ The CEO Frenkel Hamiltonian given in Table 4 is thus in good agreement with experiment (a minor rescaling factor $\left(\mu_{\text {calc }} / \mu_{\text {exp }}\right)^{2}=1.17$ will give a perfect match).

We next turn to the analysis of interchromophore energy transfer in LH2. The calculated couplings could be immediately employed to estimate B800-B800 and B800-B850 Förster energy-transfer time scales. ${ }^{33}$ The upper limit estimates of spectral overlaps in B800-B800 and B800-B850 determined 
TABLE 4: Calculated Energy Transfer Rates (Eq 1.1) between B800-B800 and B800-B850 Chromophores in the LH2 Complex of Rs. Molischianum ${ }^{a}$

\begin{tabular}{ccc}
\hline $\begin{array}{c}\text { donor-acceptor } \\
\text { transition }\end{array}$ & $\begin{array}{c}\text { calculated } \\
\text { rate }^{-1}(\mathrm{ps})\end{array}$ & $\begin{array}{c}\text { experimental } \\
\text { rate }^{-1}(\mathrm{ps})\end{array}$ \\
\hline $\mathbf{B 8 0 0}-\mathbf{B 8 0 0}$ & 0.41 & $0.8-0.9^{b}\left(0.3-0.5^{c}\right)$ \\
$1-2$ & 0.42 & \\
$1-3$ & 21.6 & \\
$\mathbf{B 8 0 0}-\mathbf{B 8 5 0}$ & 0.44 & $0.6-0.7^{d}$ \\
$1-1 \alpha$ & 10.8 & \\
$1-1 \beta$ & 1.32 & \\
$1-2 \alpha$ & 0.78 & \\
$1-2 \beta$ & 5.5 &
\end{tabular}

${ }^{a}$ The chromophore labeling and electronic coupling are shown in Figure 4. B800-B800 and B800-B850 total transfer rates are obtained by summing the rates over all possible channels. Experimental total rates are given as well. ${ }^{b}$ Reference 4 . Absorption anisotropy decay in Rs. molischianum. ${ }^{c}$ References 4, 20, and 29. Femtosecond studies of Rps. acidophila. ${ }^{d}$ References $18-20$. Femtosecond studies of $R s$. sphaeroides and Rps. acidophila.

by Pullerits et al. ${ }^{34}$ are 0.0032 and 0.0004 , respectively. By substituting the calculated electronic couplings and spectral overlaps into eq 1.1, we obtained the B800-B800 and B800B850 energy-transfer rates summarized in Table 4 . The interaction between B800 pigments is weak, and the energy-transfer rate $^{4,32}$ is properly described by the Förster mechanism. Our B800-B800 total transfer rate is in good agreement with experiment and with other calculations, since the computed couplings are similar for different approaches (see Table 3). Since the maximum possible spectral overlap ${ }^{34}$ has been used, our computed time scale $(0.41 \mathrm{ps})$ is somewhat faster than experiment.

The B800-B850 energy transfer has been the subject of an extensive debate. The Förster transfer rates calculated using 30 $\mathrm{cm}^{-1}$ for the coupling consistently predicted a longer time (e.g., $1.3 \mathrm{ps}$ in ref 32 and $1.9 \mathrm{ps}$ in ref 34$)$ than experiment $(0.6-0.7$ ps in refs 18-20). Other mechanisms (e.g., upper exciton band transfer ${ }^{4,29,34}$ and superexchange type coupling through carotenoid $^{32}$ have been suggested to explain this faster transfer. A more microscopic modeling of the rate should take into account explicitly the intramolecular vibronic structure of the lineshapes. ${ }^{67}$ For example, eq 1.1 predicts that the B800 to B850 transfer rate should be well over an order of magnitude slower at $4 \mathrm{~K}$ than at room temperature since the spectral overlap is very small. In reality it is only a factor of 3 slower. This can be accounted for once the vibronic structure is incorporated. The B800 to B850 energy transfer was simulated using experimentally measured Franck-Condon factors for intramolecular and phonon modes, the width of the homogeneous distribution of B800-B850 energy gap values, and the homogeneous width of the spectral density. ${ }^{29}$ These calculations show that BChl provides a dense picket fence of intramolecular modes. The gaps between the pickets are filled in by the phonons. Thus, one has a very wide and essentially quite constant quasicontinum of acceptor levels. This is why the temperature dependence of the B800 to B850 EET rate is weak and why it is resilient to significant pressure and mutation-induced changes in the B800B850 energy gap. The larger B800-B850 electronic couplings predicted by the CEO (see Table 3 ) reproduce the experimental Förster hopping rates without involving any additional mechanisms. Note that the other coupling constants do not generally exceed the estimates of other methods. Our computed 0.44 ps time scale is shorter than experiment. Again this may be attributed the upper-limit estimate of the spectral overlap used in our Förster transfer calculations. ${ }^{34}$
In summary, we have demonstrated that the CEO approach allows a fast and inexpensive calculation of the Frenkel exciton Hamiltonian in extended molecular aggregates as well as a realspace visualization of the various electronic excitations. Energytransfer rates in the LH2 complex of Rs. molischianum computed using this effective Hamiltonian are in good agreement with experiment.

Acknowledgment. We thank Prof. K. Shulten and Prof. M. Zerner for providing us with crystal structures of Rs. molischianum and Prof. G. Small for most useful discussions. The support of the National Science Foundation is gratefully acknowledge.

\section{References and Notes}

(1) Zuber, H.; Brunisholz, R. A. Photosynthesis: Physical Mechanism and Chemical Patterns; Cambridge University Press: New York, 1980.

(2) Zuber, H.; Brunisholz, R. A. In Chlorophylls; Scheer, H., Ed.; CRC: Boca Raton, FL, 1991; p 1047.

(3) Pullerits, T.; Sundström, V. Acc. Chem. Res. 1996, 29, 381.

(4) Pullerits, T.; Sundström, V.; van Grondelle, R. J. Phys. Chem. 1999, 103,2327

(5) Fleming, G. R.; van Grondelle, R. Phys. Today, 1994, 47, 48.

(6) Hu, X.; Damjanovic, A.; Ritz, T.; Schulten, K. Proc. Natl. Acad. Sci. U.S.A. 1998, 95, 5935 .

(7) van Grondelle, R.; Deckker, J. P.; Gillbro, T.; Sundström, Biochim. Biophys. Acta 1994, 1187, 1.

(8) Sundström, V.; van Grondelle, R. In Anoxigenic Photosynthetic Bacteria; Blankenship, R. E., Madiga, M. T., Baner, C. E., Eds.; Kluwer Academic: Drodrecht, 1995; p 349.

(9) Hu, X.; Schulten, K. Phys. Today 1997, 50, 28.

(10) Miller, K. Nature 1982, 300, 53.

(11) Karrasch, S.; Bullough, P. A.; Gosh, R. EMBO J. 1995, 14, 631. 614 .

(12) Kuhlbrandt, W.; Wang, D. N.; Fujiyoshi, Y. Nature, 1994, 367,

(13) Savage, H.; Cyrklaff, M.; Montoya, G.; Kuhlbrandt, W.; Sinning, I. Structure, 1996, 4, 243 .

(14) McDermott, G.; Prince, S. M.; Freer, A. A.; HawthornthwaiteLawless, A. M.; Papiz, M. Z.; Cogdell, R. J.; Isaacs, N. W. Nature, 1995, $374,517$.

(15) Koepke, J.; Hu, X.; Muenke, C.; Schulten, K.; Michel, H. Structure 1996, 4, 581.

(16) Sauer, K.; Cogdell, R. J.; Prince, S. M.; Freer, A. A.; Isaacs, N. W.; Scheer, H. Photochem. Photobiol. 1996, 64, 564

(17) Alden, R. G.; Johnson, E.; Nagarajan, V.; Parson, W. W.; Law, C. J.; Cogdell, R. G. J. Phys. Chem. B 1997, 101, 4667.

(18) Shreve, A. P.; Trautman, J. K.; Frank, H. A.; Owens, T. G.; Albrecht, A. C. Biochim. Biophys. Acta 1991, 280, 1058.

(19) Ma, Y.-Z.; Cogdell, R. J.; Gillbro, T. J. J. Phys. Chem. B 1997, 101,1087

(20) Ma, Y.-Z.; Cogdell, R. J.; Gillbro, T. J. J. Phys. Chem. B 1998 , 102,881

(21) Joo, T.; Jia, Y.; Yu, J.-Y.; Jonas, D. M.; Fleming, G. R. J. Phys Chem. 1996, 100, 2399.

(22) Reddy, N. R. S.; Picorel, R.; Small, G. J. J. Phys. Chem. 1992, 96, 9458.

(23) Pullerits, T.; Chachivilis, M.; Sundström, V. J. Phys. Chem. 1996, 100,10787

(24) Hess, S.; Feldchtein, F.; Babin, A.; Nurgaleev, I.; Pullerits, T.; Sergeev, A.; Sundström, V. Chem. Phys. Lett. 1993, 216, 247.

(25) Monshouwer, R.; de Zarate, I. O.; van Mourik, F.; van Grondelle, R. Chem. Phys. Lett. 1995, 246, 341

(26) Chachivilis, M.; Fidder, H.; Pullerits, T.; Sundström, V. J. Raman Spectrosc. 1995, 26, 513

(27) Chachivilis, M.; Pullerits, T.; Jones, M. R.; Hunter, C. N.; Sundström, V. J. Phys. Chem. B 1997, 101, 7275.

(28) Nagarajan, V.; Alden, R. G.; Williams, J. C.; Parson, W. W. Proc. Natl. Acad. Sci. U.S.A., 1996, 93, 13774.

(29) Wu, H.-M.; Savikhin, S.; Reddy, N. R. S.; Jankowiak, R.; Cogdell, R. J.; Struve, W. S.; Small, G. J. J. Phys. Chem. 1996, 100, 12022.

(30) Kumble, R.; Hochstrasser, R. J. Chem. Phys. 1998, 109, 855

(31) Jimenez, R.; van Mourik, F.; Fleming, G. R. J. Phys. Chem. B 1997, 101,7350

(32) Krueger, B. P.; Scholes, G. D.; Fleming, G. R. J. Phys. Chem. B 1998, 102, 5378 .

(33) Förster, Th. Naturwissenschaften 1946, 33, 166.

(34) Pullerits, T.; Hess, S.; Herek, J. L.; Sundström, V. J. Phys. Chem. B 1997, 101, 10560 . 
(35) Meier, T.; Chernyak, V.; Mukamel, S. J. Phys. Chem. B 1997, 101, 7332.

(36) Meier, T.; Zhao, Y.; Chernyak, V.; Mukamel, S. J. Chem. Phys. 1997, 107, 1.

(37) Pearlstein, R. M. In Chlorophylls; Scheer, H., Ed.; CRC: Boca Raton, FL, 1991; 1047.

(38) Scholes, G. D.; Gould, I. R.; Cogdell, R. J.; Fleming, G. R. J. Phys. Chem. B 1999, 103, 2543.

(39) Krueger, B. P.; Scholes, G. D.; Jiminez, R.; Fleming, G. R. J. Phys. Chem. B 1998, 102, 2284.

(40) Linnanto, J.; Korppi-Tommola, J. E. I.; Helenius, V. M. J. Phys. Chem B. 1999, 103, 8739.

(41) Koolhaas, M. H. C.; Frese, R. N.; Fowler, G. J. S.; Bibby, T. S . Georgakopoulou, S.; van der Zwan, G.; Hunter, C. N.; van Grondelle, R. Biochemistry 1998, 14, 4693.

(42) Hu, X.; Ritz, T.; Damjanovic, A.; Schulten, K. J. Phys. Chem. 1997, 101,3854 .

(43) Cory, M. G.; Zerner, M. C.; Hu, X.; Schulten, X. K.; J. Phys. Chem. 1998, 102, 7640.

(44) Tretiak, S.; Chernyak, V.; Mukamel, S. Chem. Phys. Lett. 1996, 259, 55. Tretiak, S.; Chernyak, V.; Mukamel, S. J. Chem. Phys. 1996, 105, 8914.

(45) Tretiak, S.; Chernyak, V.; Mukamel, S. J. Am. Chem. Soc. 1997, 119,11408 .

(46) Mukamel, S.; Tretiak, S.; Wagersreiter, T.; Chernyak, V. Science 1997, 277, 781

(47) Tretiak, S.; Chernyak, V.; Mukamel, S. J. Phys. Chem. B 1998, 102,3310 .

(48) Tretiak, S.; Zhang, W. M.; Chernyak, V.; Mukamel, S. Proc. Nat. Acad. Sci. 1999, 96, 13003.

(49) Bazan, G. C.; Oldham, W. J., Jr.; Lachicotte, R. J.; Tretiak, S.; Chernyak, V.; Mukamel, S. J. Am. Chem. Soc. 1998, 120, 9188.

(50) Tretiak, S.; Chernyak, V.; Mukamel, S. Chem. Phys. Lett. 1998, 297, 357.

(51) Poliakov, E.; Chernyak, V.; Tretiak, S.; Mukamel, S. J. Chem. Phys. 1999, 110, 8161. Minami, T.; Tretiak, S.; Chernyak, V.; Mukamel, S. J. Lumin., in press.

(52) Pople, J. A.; Segal, G. A. J. Chem. Phys. 1965, 43, S136.
(53) Pople, J. A.; Beveridge, D. L.; Dobosh, P. J. Chem. Phys. 1967, 47, 2026.

(54) Ridley, J.; Zerner, M. C. Theor. Chim. Acta 1973, 32, 111.

(55) Zerner, M. C.; Loew, G. H.; Kirchner, R. F.; Mueller-Westerhoff, U. T. J. Am. Chem. Soc. 1980, 102, 589.

(56) McWeeny, R.; Sutcliffe, B. T. Methods of Molecular Quantum Mechanics; Academic Press: New York, 1976.

(57) Davidson, E. R. Reduced Density Matrices in Quantum Chemistry; Academic Press: New York, 1976.

(58) Previously we have used the density matrix spectral moment algorithm for computing the eigenmodes. ${ }^{44}$ That algorithm is particularly suitable for off-resonant static response computations where only transitions with nonzero oscillator strengths are calculated. We found that the oblique Lanczos algorithm is more efficient and provides a more accurate representation of the resonant response than the DSMA.

(59) Chernyak, V.; Tretiak, S.; Schulz, M.; Tsiper, E. V.; Mukamel, S. Submitted for publication in J. Chem. Phys.

(60) Oelze, J. Methods Microbiol. 1985, 18, 257

(61) Pope, M.; Swenberg, C. E. Electronic Processes in Organic Crystals; Clarendon Press, Oxford University Press: Oxford, New York, 1982.

(62) E. A. Silinsh, Čápek, V. Organic Molecular Crystals; AIP Press, American Institute of Physics: New York, 1994. 1971.

(64) Kasha, M.; Rawls, H. R.; Ashraf El-Bayoumi, M. Pure Appl. Chem. $1965,11,371$

(65) Rashba, E. I.; Sturge, M. D., Eds. Excitons; North-Holland: Amsterdam, 1982. Broude, V. B.; Rashba, E. I.; Sheka, E. F. Spectroscopy of Molecular Excitons; Springer: Berlin, 1985.

(66) Baker, J. D.; Zerner, M. C.; Chem. Phys. Lett. 1990, 175, 192.

(67) Small, G. J.; Hayes, J. M.; Silbey, R. J. J. Phys. Chem. 1992, 96 7499. Kolaczkowski, S. V.; Hayes, J. M.; Small, G. J. J. Phys. Chem. 1994, 98, 13418.

(68) For a general discussion of this representation, see eq 4.36 in Mukamel, S. Principles of Nonlinear Optical Spectroscopy; Oxford: New York, 1995. 